\title{
Entropic Lower Bound for Distinguishability of Quantum States
}

\author{
Seungho Yang, ${ }^{1}$ Jinhyoung Lee, ${ }^{1,2}$ and Hyunseok Jeong ${ }^{1}$ \\ ${ }^{1}$ Center for Macroscopic Quantum Control, Department of Physics and Astronomy, Seoul National University, \\ Seoul 151-742, Republic of Korea \\ ${ }^{2}$ Department of Physics, Hanyang University, Seoul 133-791, Republic of Korea
}

Correspondence should be addressed to Hyunseok Jeong; h.jeong37@gmail.com

Received 18 August 2015; Accepted 22 October 2015

Academic Editor: Remi Léandre

Copyright (C) 2015 Seungho Yang et al. This is an open access article distributed under the Creative Commons Attribution License, which permits unrestricted use, distribution, and reproduction in any medium, provided the original work is properly cited.

For a system randomly prepared in a number of quantum states, we present a lower bound for the distinguishability of the quantum states, that is, the success probability of determining the states in the form of entropy. When the states are all pure, acquiring the entropic lower bound requires only the density operator and the number of the possible states. This entropic bound shows a relation between the von Neumann entropy and the distinguishability.

\section{Introduction}

Quantum mechanics does not allow determining the state of a system by measuring a single copy of the ensemble. Nevertheless, if some prior information is known, it is possible to guess the state with a certain degree of confidence even by a single measurement. Given some prior information, to what extent quantum states can be distinguished is an intriguing issue from both fundamental and practical points of view. For example, this problem is closely related to efficiencies of quantum communication [1-3]. It is known that the imperfect distinguishability plays a crucial role in the security of quantum cryptography [3]

There are different approaches to the distinguishability of quantum states [4-7]. In the minimum-error discrimination problem [4], a set of known quantum states and preparation probabilities are given, and one aims to distinguish the states with the optimal probability of success. The optimal success probability is an operationally well-defined measure for the distinguishability of given states. However, it is a highly demanding quest to find its analytical solution for general sets of states, and the solution is only known for the sets of two states [1]. Instead, upper bounds [8-12] and lower bounds [13-15] have been provided to estimate the optimal probability. On the other hand, there have been studies of distinguishability between unknown quantum states using programmable machines [6, 7]. In this case, ancillary systems prepared in each of the unknown states are provided as an input of the machine.

One may pay attention to the von Neumann entropy as a quantity related to the distinguishability in light of the capacity of a quantum state for embodying quantum information. When a system is probabilistically prepared in one of a certain number of quantum states, its state of the statistical mixture is described by a density operator. According to the quantum source theorem [16], the von Neumann entropy of the system, which is given as a function of the density operator, represents the capacity of the mixed state to (asymptotically) carry quantum information. As discussed in [17], one may relate this capacity to the concept of distinguishability because more information could be carried when each state is more distinguishable. For this reason, Jozsa and Schlienz considered the von Neumann entropy as measure for distinguishability [17]. However, it is not known whether this kind of distinguishability is linked to the actual ability to distinguish quantum states by measurements, namely, the success probability (distinguishability will henceforth refer to the success probability). It seems that, at least, the von Neumann entropy cannot pinpoint the success probability of distinguishing given quantum states. This is because the von Neumann entropy is determined only by the density operator, and the density operator of a system can take 
arbitrarily many decompositions in general; thus it does not contain information on which states the system could have been prepared in.

We here present a lower bound for the distinguishability, that is, the optimal success probabilities of distinguishing between quantum states, as a function of entropies of the system. For a system prepared in one of $N$ pure states, we also present a reduced form of the entropic bound which requires the density operator and the number of possible states $N$ for its evaluation. It reveals a relation between the von Neumann entropy and the distinguishability; the larger von Neumann entropy guarantees the better distinguishability.

\section{General Formulation}

Consider a quantum system prepared in one of $N$ quantum states with some probabilities. We denote them by $\left\{p_{x}, \rho_{x}\right\}_{x=1}^{N}$. One wishes to identify which state the system has been prepared in or, equivalently, to identify the value of $x$. The value of $x$ is determined, using a generalized measurement described by the measurement operator $\left\{\widehat{M}_{x}\right\}_{x=1}^{N}$. Therefore, the probability of correctly identifying $x$ for a given $x$ is $\operatorname{Tr}\left[\widehat{M}_{x} \rho_{x}\right]$, and the expected success probability is

$$
P_{s}=\sum_{x=1}^{N} p_{x} \operatorname{Tr}\left(\widehat{M}_{x} \rho_{x}\right) .
$$

When maximized over all measurements, it becomes the optimal success probability, which is denoted by $P_{s}^{*}$. It is the quantity which we consider as the degree of the distinguishability of quantum states. As already mentioned, however, the analytical form of $P_{s}^{*}$ is known only for the twostate case [1].

An equivalent way of describing the scenario is to consider a classical-quantum system $X Q$ in the state,

$$
\sigma_{X Q}=\sum_{x} p_{x}|x\rangle\langle x| \otimes \rho_{x}
$$

where the indices $x$ 's are encoded. Namely, one is given the quantum system $Q$ and wishes to determine the value of $x$ by measuring $Q$. In terms of entropic quantities, $X$ has uncertainty quantified by Shannon entropy $H(X)=$ $-\sum p_{x} \log p_{x}$, and it has a correlation with the quantum system.

One may expect an entropic lower bound from the intuition that the correlation of $Q$ with $X$ would enhance the distinguishability of the quantum states. For better understanding, let us first consider a fully classical case [18], where the quantum system $Q$ is replaced with a classical system $Y$ and $\rho_{x}=\sum_{y} p(y \mid x)|y\rangle\langle y|$. Assume that we are given $Y$ and wish to determine the value of $x$ from $y$. For given $y$, the most probable $x$ is the one that gives the maximum conditional probability, $\max _{x} p(x \mid y)$. Therefore, the optimal success probability is attained by choosing them for all $y$, and it is given as $P_{s}^{*}=\sum_{y} p(y) \max _{x} p(x \mid y)$. It can be lower bounded in terms of the correlation between $X$ and $Y$ as follows:

$$
\begin{aligned}
P_{s}^{*} & =\sum_{y} p(y) \max _{x}(x \mid y) \geqslant \sum_{y} p(y) \sum_{x} p(x \mid y)^{2} \\
& =\sum_{x, y} p(x, y) p(x \mid y)=\sum_{x, y} p(x, y) 2^{\log p(x \mid y)} \\
& \geqslant 2^{\sum_{x, y} p(x, y) \log p(x \mid y)} .
\end{aligned}
$$

The first inequality follows by taking the average of $p(x \mid y)$ over $p(x \mid y)$, and the second one follows from the concavity of the exponential function. The exponent in the last line is equal to the conditional Shannon entorpy $H(X \mid Y)$ so that

$$
P_{s}^{*} \geqslant 2^{-H(X \mid Y)}=2^{-H(X)+H(X: Y)},
$$

where $H(X: Y)$ is the classical mutual information between $X$ and $Y$. Therefore, with assistance from the random variable $Y$ having the amount of correlation $H(X: Y)$, one can guess the random variable $X$ with probability (in logarithm) at least $-H(X)+H(X: Y)$.

\section{Entropic Lower Bound for the Distinguishability of Quantum States}

For the quantum case, we can still obtain a random variable $\tilde{Y}$ by applying a measurement (i.e., the outcome of the measurement can be considered as random variable), and applying (4) gives $\log P_{s}^{*} \geqslant-H(X \mid \tilde{Y})$. However, this bound can be further sharpened by using the quantum entropies. For a quantum system prepared in a density operator $\chi_{A}$, the von Neumann entropy $S(A)$ is defined as $S(A)=S\left(\chi_{A}\right)=$ $-\operatorname{Tr}\left[\chi_{A} \log \chi_{A}\right]$. The conditional von Neumann entropy of a bipartite system $A B$ is defined as $S(A \mid B)=S(A B)-S(B)$. Similarly, $I(A: B)=S(A)+S(B)-S(A B)$ defines the von Neumann mutual information [19]. In terms of the quantum entropies, we present the quantum entropic lower bound.

Theorem 1. For a set of quantum states with preparation probabilities $\left\{p_{x}, \rho_{x}\right\}_{x=1}^{N}$, the optimal success probability of distinguishing the quantum states, $P_{s}^{*}$, is lower bounded as

$$
\begin{aligned}
P_{s}^{*} & \geqslant 2^{-S(X \mid Q)}=2^{-H(X)+I(X: Q)} \\
& =2^{-H(\vec{p})+S\left(\sum p_{x} \rho_{x}\right)-\sum p_{x} S\left(\rho_{x}\right) .}
\end{aligned}
$$

Proof. For the proof, we employ the conditional min-entropy [20], which has many applications in quantum cryptography. The conditional min-entropy $S_{\min }(A \mid B)$ of a system $A B$ in a state $\rho_{A B}$ is defined as

$$
\begin{aligned}
S_{\min } & (A \mid B) \\
= & -\inf _{\sigma_{B} \in \pi_{B}} \inf \left\{\lambda \in \mathbb{R}: \rho_{A B} \leqslant 2^{\lambda}\left(\mathbb{1}_{A} \otimes \sigma_{B}\right)\right\},
\end{aligned}
$$

where $\pi_{B}$ denotes the set of all quantum states of the subsystem $B$. We derive the lower bound (5) from two properties 
of the conditional min-entropy. First, the conditional minentropy has an operational meaning that, for the classicalquantum states in (2), the logarithm of $P_{s}^{*}$ is equal to the negative conditional min-entropy (see [21] for the details). Therefore, for the classical-quantum state in (2),

$$
\log P_{s}^{*}=-S_{\min }(X \mid Q) \text {. }
$$

It has also been shown in [22] that the conditional minentropy is always less than or equal to the conditional von Neumann entropy, so we have

$$
S_{\min }(A \mid B) \leqslant S(A \mid B) .
$$

Using (7) and (8), we obtain

$$
\log P_{s}^{*} \geqslant-S(X \mid Q),
$$

which is equivalent to the first line in (5). On the other hand, the conditional von Neumann entropy satisfies the chain rule, $S(X \mid Q)=S(X)-S(X Q)$. It enables the lower bound to be written as a function of entropies of the system $Q$. The von Neumann entropies of $X Q$ and $X$ are evaluated as $S(X Q)=$ $S\left(\sum p_{x} \rho_{x}\right)-\sum p_{x} S\left(\rho_{x}\right)$ and $S(X)=H(\vec{p})$. It then follows that $S(X \mid Q)=H(\vec{p})-S\left(\sum p_{x} \rho_{x}\right)+\sum p_{x} S\left(\rho_{x}\right)$, and this gives the second line in (5).

Let us take a closer look into the form of the lower bound. Its form is exactly of (4), but only $H(X: Y)$ is replaced with $I(X: Q)$. The quantum mutual information is known to capture all the correlations including both classical and quantum parts $[23,24]$, so it is considered as a measure of the total correlations. Hence, we see that the lower bound is increased by the total correlation. For the classical-quantum state $\sigma_{X Q}$, we obtain $I(X: Q)=S\left(\sum p_{x} \rho_{x}\right)-\sum p_{x} S\left(\rho_{x}\right)$, and Holevo's theorem $[25,26]$ implies that $H(X: \tilde{Y}) \leqslant$ $S\left(\sum p_{x} \rho_{x}\right)-\sum p_{x} S\left(\rho_{x}\right)=I(X: Q)$ for any measurement on the system $Q(\tilde{Y}$ is the outcome of a measurement on $Q)$. The minimum difference between $I(X: Q)$ and $H(X: \tilde{Y})$, that is, $I(X: Q)-\max H(X: \widetilde{Y})$, is equal to quantum discord [27] of $\sigma_{X Q} \cdot$

On the assumption that the system is prepared in one of $N$ pure states $\left\{p_{x}, \psi_{x}\right\}_{x=1}^{N}$, the entropic bound can be reduced to a form that only requires the density operator of the system and the number of the possible states for its evaluation. Using $H(\vec{p}) \leqslant \log N$ and $S\left(\psi_{x}\right)=0$, we have

$$
P_{s}^{*} \geqslant \frac{2^{S\left(\sum p_{x}\left|\psi_{x}\right\rangle\left\langle\psi_{x}\right|\right)}}{N} .
$$

Therefore, we see that the larger von Neumann entropy guarantees the better distinguishability. Notwithstanding the missing information on the component states and the preparation probabilities, the density operator of a system alone can provide a lower bound for distinguishability of $N$ pure states. Note that when many copies of quantum systems are prepared in the same way, the density operator can be obtained using the state tomography, but it is impossible to guess the component states. The state-discrimination machine with unknown quantum states as an input $[6,7]$ is the case where it is required to distinguish between unknown quantum states.

\section{Other Lower Bounds and Examples}

In this section, we compare the entropic lower bound to other previously known bounds. One is the lower bound given by the square-root measurement [13], and it is known to be optimal for many cases in which the solutions are known [4]. The measurement operators of the square-root measurement $\left\{\pi_{i}\right\}_{i=1}^{N}$ are given as

$$
\widehat{\pi}_{x}=p_{x} \rho^{-1 / 2} \rho_{x} \rho^{-1 / 2},
$$

where $\rho=\sum_{x} p_{x} \rho_{x}$. Therefore, a lower bound by the squareroot measurement is given as

$$
P_{s}^{*} \geqslant \sum_{x=1}^{N} p_{x} \operatorname{Tr}\left(\rho_{x} \widehat{\pi}_{x}\right) .
$$

Another one is the pairwise-overlap bound. For an ensemble of pure states $\left\{p_{x}, \psi_{x}\right\}_{x=1}^{N}$, it has been given in [14] as

$$
P_{s}^{*} \geqslant \sum_{i=1}^{N} \frac{p_{i}^{2}}{\sum_{j=1}^{N} p_{j}\left|\left\langle\psi_{i} \mid \psi_{j}\right\rangle\right|^{2}} .
$$

This was derived as a lower bound for the square-root measurement bound, so it is always less than or equal to the bound by the square-root measurement. It provides an analytic form of lower bounds in terms of the pairwise overlaps.

We now consider a few exemplary sets of pure states $\left\{\psi_{x}\right\}_{x=1}^{N}$ and compare the entropic bound in (10) with the other two lower bounds. Let us first look at three 3dimensional pure states with equal probabilities:

$$
\begin{aligned}
& \left|\psi_{1}\right\rangle=\sin \theta|0\rangle+\cos \theta|2\rangle, \\
& \left|\psi_{2}\right\rangle=|1\rangle, \\
& \left|\psi_{3}\right\rangle=-\sin \theta|0\rangle+\cos \theta|2\rangle .
\end{aligned}
$$

The entropic lower bound and the other two bounds are calculated and plotted in Figure 1(a). In Figure 1(b), we present the bound values with another set of states, where only $\left|\psi_{2}\right\rangle$ is replaced with $(|0\rangle+|1\rangle) / \sqrt{2}$ from the previous case. As shown in Figure 1, for both the sets of states, the square-root measurement provides the tightest bounds. The entropic bound is shown to be greater than the pairwiseoverlap bound for large regions.

The next example of the component states is four 2dimensional states with equal probabilities,

$$
\begin{aligned}
& \left|\psi_{1}\right\rangle=|0\rangle, \\
& \left|\psi_{2}\right\rangle=\sin \theta|0\rangle+\cos \theta|1\rangle, \\
& \left|\psi_{3}\right\rangle=|1\rangle, \\
& \left|\psi_{4}\right\rangle=\cos \theta|0\rangle-\sin \theta|1\rangle,
\end{aligned}
$$

which satisfy $\left\langle\psi_{1} \mid \psi_{3}\right\rangle=\left\langle\psi_{2} \mid \psi_{4}\right\rangle=0$. In this case, the optimal success probability is known as $1 / 2$ for any $0 \leqslant$ 


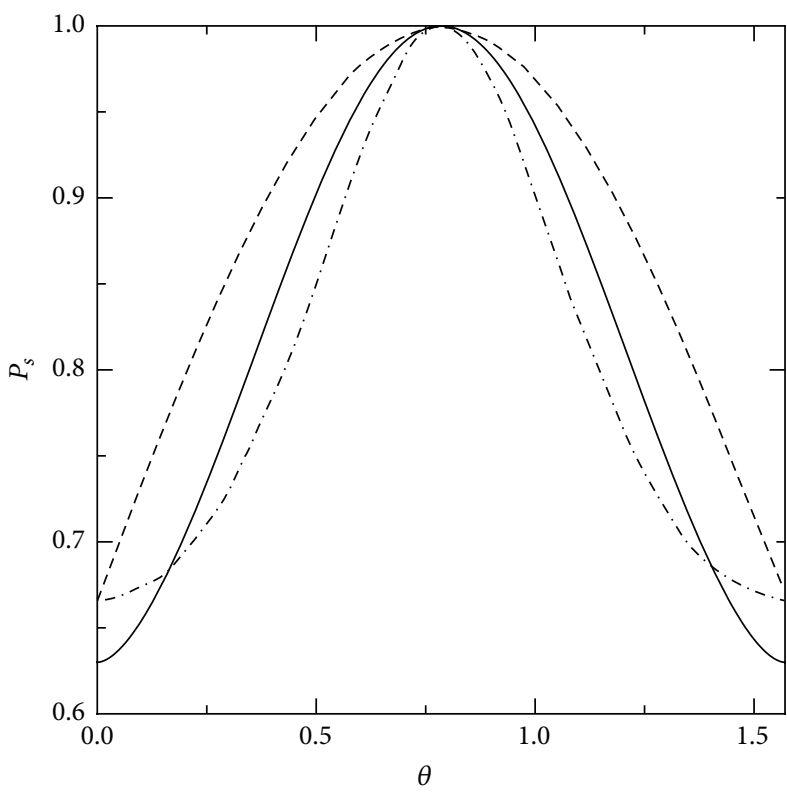

(a)

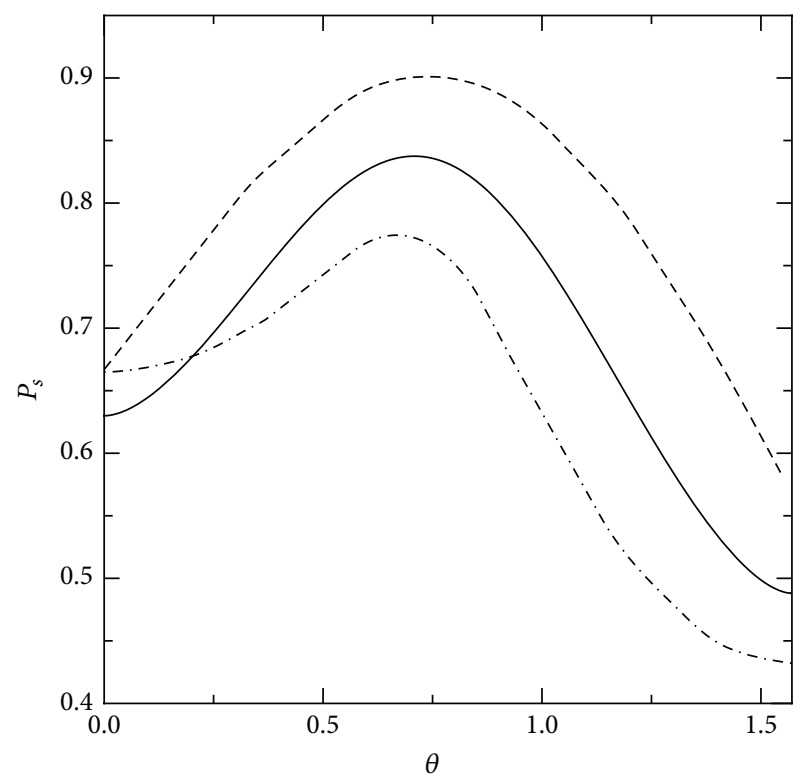

(b)

FIGURE 1: (a) Lower bounds for the optimal discrimination probability for the states in (14). (b) Lower bounds for the same set but with $\left|\psi_{2}\right\rangle=$ $(|0\rangle+|1\rangle) / 2$. The entropic lower bounds are plotted as the solid curves. For comparison, the lower bounds by the square-root measurement and the pairwise-overlap bounds are represented by the dashed and dot-dashed curves, respectively.

$\theta \leqslant 2 \pi[28]$. The entropic bound gives $1 / 2$, and the other two lower bounds also give $1 / 2$.

Finally, we consider a discrimination problem where the component states are not given, but the density operator and the number of possible states are given. Assume that a system is prepared in one of $N$-dimensional unknown states, but the system is described by the density operator $\rho=\mathbb{1} / n$. There are arbitrarily many possibilities of choosing component states and their preparation probabilities in constructing the density operator. For instance, in the case where $N=4$ and $n=2$, we see that the four states in (15) with $p_{1}=p_{3}=q / 2$ and $p_{2}=p_{4}=(1-q) / 2$ give rise to the same density operator $\rho=\mathbb{1} / 2$ for any $0 \leqslant q \leqslant 1$ and $0 \leqslant \theta \leqslant 2 \pi$. In this case, the square-root measurement cannot be specified, so one needs to take a minimization over all decompositions of the density operator to obtain a lower bound. However, the entropic bound provides $P_{s}^{*} \geqslant N^{-1} 2^{S(\mathbb{1} / n)}=1 / 2$ regardless of the component states and the preparation probabilities.

\section{Conclusion}

We have presented an entropic lower bound for the optimal success probability of distinguishing quantum states. It provides a connection between the optimal discrimination probability and quantum entropy, that is, between a practically relevant quantity and a primary function in quantum information theory. When the quantum states are all pure, the entropic bound is reduced to a form that requires less information for its evaluation, namely, the density operator and the number of the possible states. It shows that the von Neumann entropy can lower bound the distinguishability of $N$ pure states.

\section{Conflict of Interests}

The authors declare that there is no conflict of interests regarding the publication of this paper.

\section{Acknowledgments}

This work was supported by the National Research Foundation of Korea (NRF) grant funded by the Korea government (MSIP) (no. 2010-0018295) and the Center for Theoretical Physics at Seoul National University.

\section{References}

[1] C. W. Helstrom, Quantum Detection and Estimation Theory, Academic Press, New York, NY, USA, 1976.

[2] A. S. Holevo, Statistical Structure of Quantum Theory, Springer, Berlin, Germany, 2000.

[3] V. Scarani, H. Bechmann-Pasquinucci, N. J. Cerf, M. Dušek, N. Lütkenhaus, and M. Peev, "The security of practical quantum key distribution," Reviews of Modern Physics, vol. 81, no. 3, pp. 1301-1350, 2009.

[4] S. M. Barnett and S. Croke, "Quantum state discrimination," Advances in Optics and Photonics, vol. 1, no. 2, pp. 238-278, 2009.

[5] K. M. R. Audenaert, J. Calsamiglia, R. Muñoz-Tapia et al., "Discriminating states: the quantum Chernoff bound," Physical Review Letters, vol. 98, no. 16, Article ID 160501, 2007.

[6] J. A. Bergou and M. Hillery, "Universal programmable quantum state discriminator that is optimal for unambiguously distinguishing between unknown states," Physical Review Letters, vol. 94, no. 16, Article ID 160501, 2005. 
[7] M. Dušek and V. Bužek, "Quantum-controlled measurement device for quantum-state discrimination," Physical Review A, vol. 66, no. 2, Article ID 022112, 2002.

[8] M. Hayashi, A. Kawachi, and H. Kobayashi, "Quantum measurements for hidden subgroup problems with optimal sample complexity," Quantum Information and Computation, vol. 8, no. 3-4, pp. 0345-0358, 2008.

[9] A. Montanaro, "A lower bound on the probability of error in quantum state discrimination," in Proceedings of the IEEE Information Theory Workshop (ITW '08), pp. 378-380, Porto, Portugal, May 2008.

[10] D. Qiu, "Minimum-error discrimination between mixed quantum states," Physical Review A, vol. 77, Article ID 012328, 2008.

[11] D. Qiu and L. Li, "Minimum-error discrimination of quantum states: bounds and comparisons," Physical Review A, vol. 81, no. 4, Article ID 042329, 2010.

[12] J. Tyson, "Two-sided estimates of minimum-error distinguishability of mixed quantum states via generalized HolevoCurlander bounds," Journal of Mathematical Physics, vol. 50, no. 3, Article ID 032106, 2009.

[13] A. S. Kholevo, "On asymptotically optimal hypothesis testing in quantum statistics," Theory of Probability \& Its Applications, vol. 23, no. 2, pp. 411-415, 1979.

[14] A. Montanaro, "On the distinguishability of random quantum states," Communications in Mathematical Physics, vol. 273, no. 3, pp. 619-636, 2007.

[15] H. Barnum and E. Knill, "Reversing quantum dynamics with near-optimal quantum and classical fidelity," Journal of Mathematical Physics, vol. 43, no. 5, pp. 2097-2106, 2002.

[16] B. Schumacher, "Quantum coding," Physical Review A, vol. 51, no. 4, pp. 2738-2747, 1995.

[17] R. Jozsa and J. Schlienz, "Distinguishability of states and von Neumann entropy," Physical Review. A, vol. 62, no. 1, Article ID 012301, 2000

[18] A. Nayak and J. Salzman, "Limits on the ability of quantum states to convey classical messages," Journal of the ACM, vol. 53, no. 1, pp. 184-206, 2006.

[19] C. Adami and N. J. Cerf, "von Neumann capacity of noisy quantum channels," Physical Review A, vol. 56, no. 5, pp. 34703483, 1997.

[20] R. Renner and S. Wolf, "Smooth rényi entropy and applications," in Proceedings of the IEEE International Symposium on Information Theory (ISIT '04), p. 232, July 2004.

[21] R. Koenig, R. Renner, and C. Schaffner, "The operational meaning of min- and max-entropy," IEEE Transactions on Information Theory, vol. 55, no. 9, pp. 4337-4347, 2009.

[22] M. Tomamichel, R. Colbeck, and R. Renner, "A fully quantum asymptotic equipartition property," IEEE Transactions on Information Theory, vol. 55, no. 12, pp. 5840-5847, 2009.

[23] L. Henderson and V. Vedral, "Classical, quantum and total correlations," Journal of Physics. A. Mathematical and General, vol. 34, no. 35, pp. 6899-6905, 2001.

[24] B. Groisman, S. Popescu, and A. Winter, "Quantum, classical, and total amount of correlations in a quantum state," Physical Review. A, vol. 72, no. 3, Article ID 032317, 2005.

[25] A. S. Holevo, "Statistical problems in quantum physics," in Proceedings of the Second Japan-USSR Symposium on Probability Theory, G. Maruyama and J. V. Prokhorov, Eds., vol. 330 of Lecture Notes in Mathematics, pp. 104-119, Springer, Berlin, Germany, 1973.
[26] M. A. Nielsen and I. L. Chuang, Quantum Computation and Quantum Information, Cambridge University Press, Cambridge, UK, 2000.

[27] H. Ollivier and W. H. Zurek, "Quantum discord: a measure of the quantumness of correlations," Physical Review Letters, vol. 88, no. 1, Article ID 017901, 2001.

[28] J. Bae, "Structure of minimum-error quantum state discrimination," New Journal of Physics, vol. 15, Article ID 073037, 2013. 


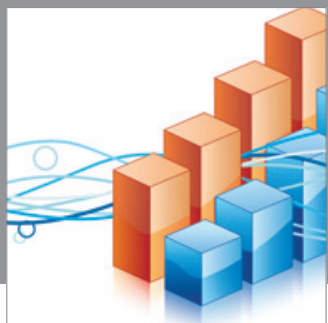

Advances in

Operations Research

mansans

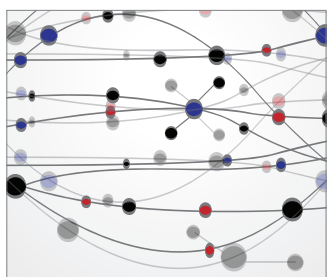

The Scientific World Journal
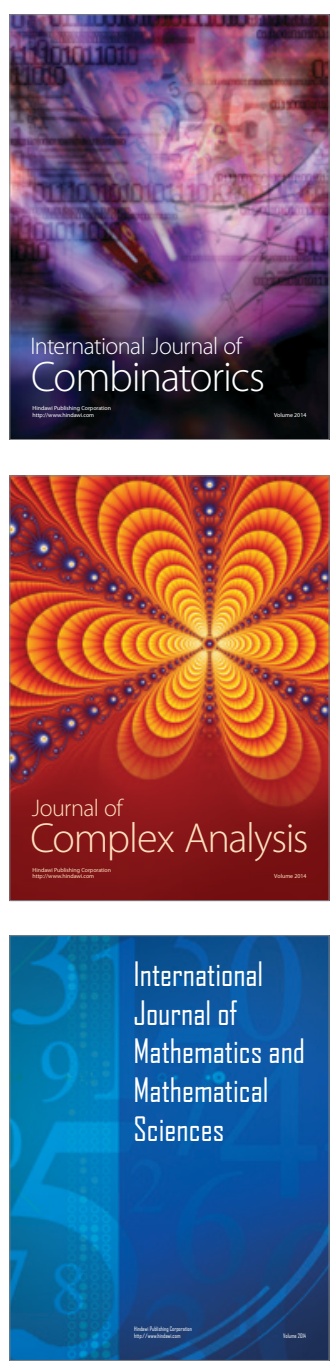
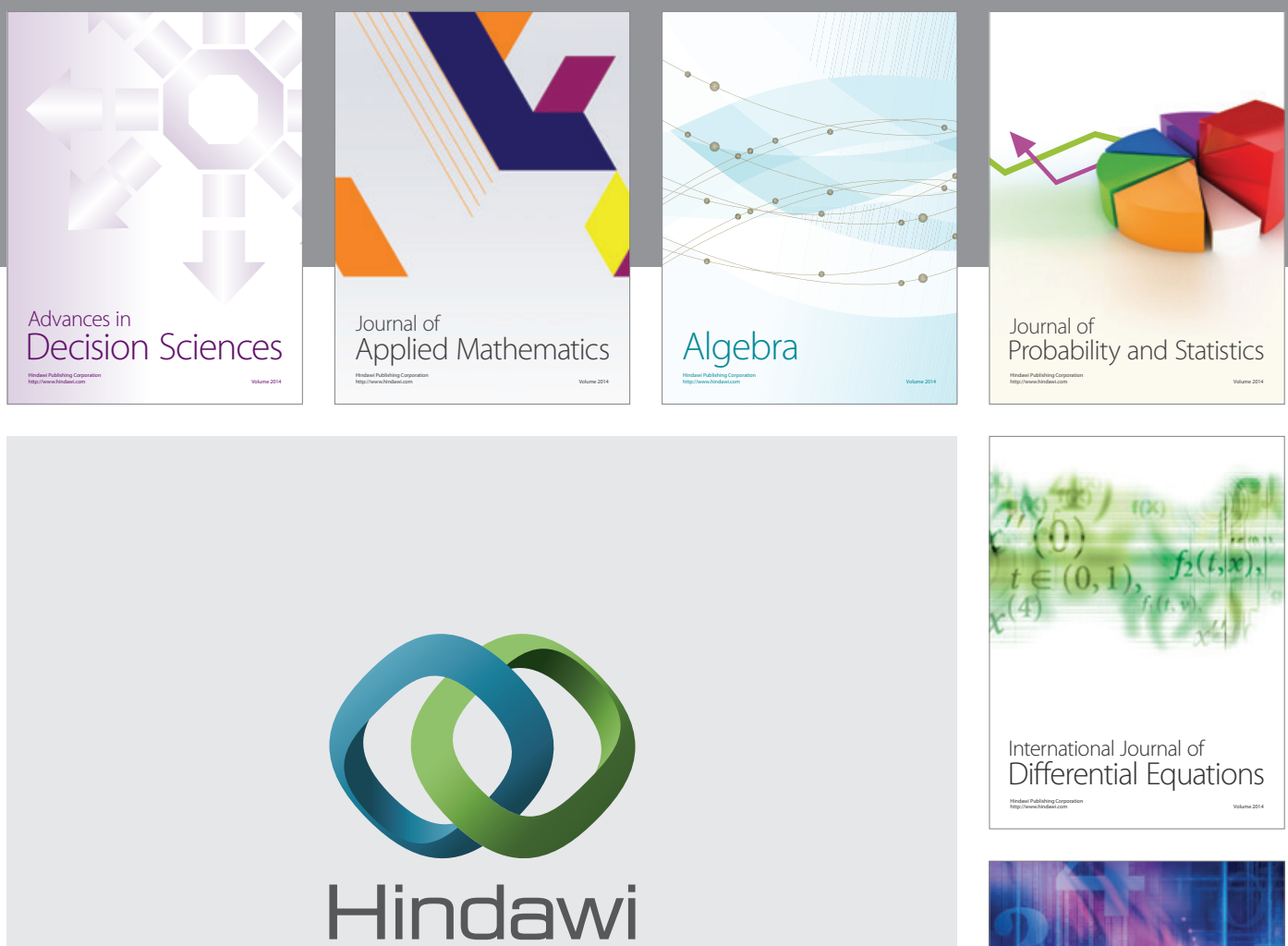

Submit your manuscripts at http://www.hindawi.com
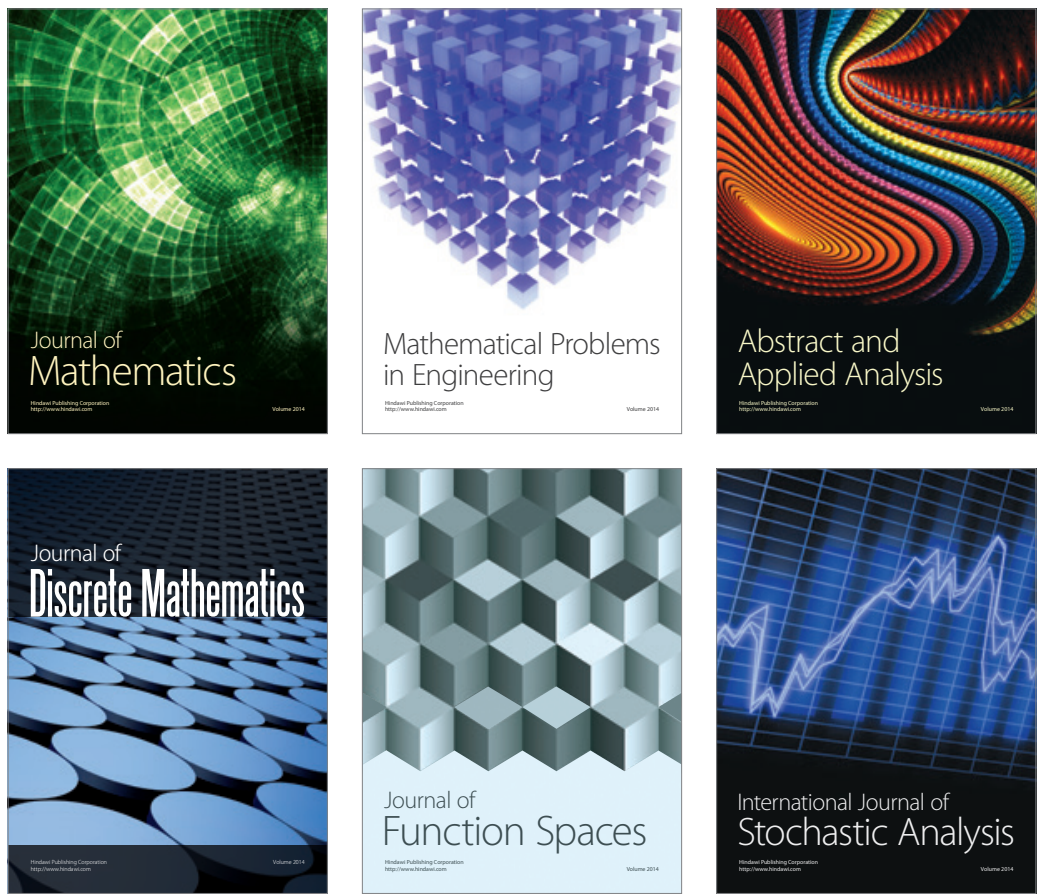

Journal of

Function Spaces

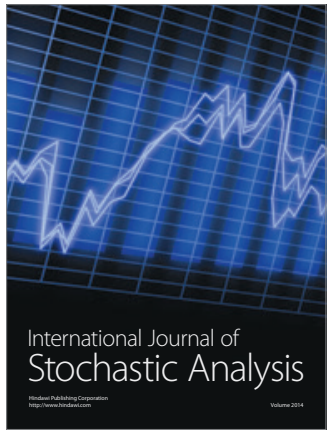

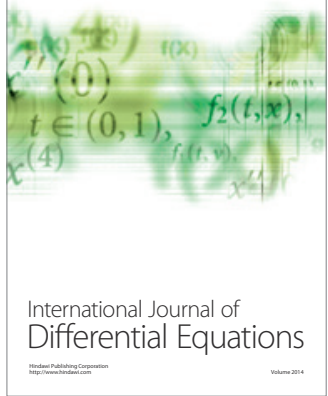
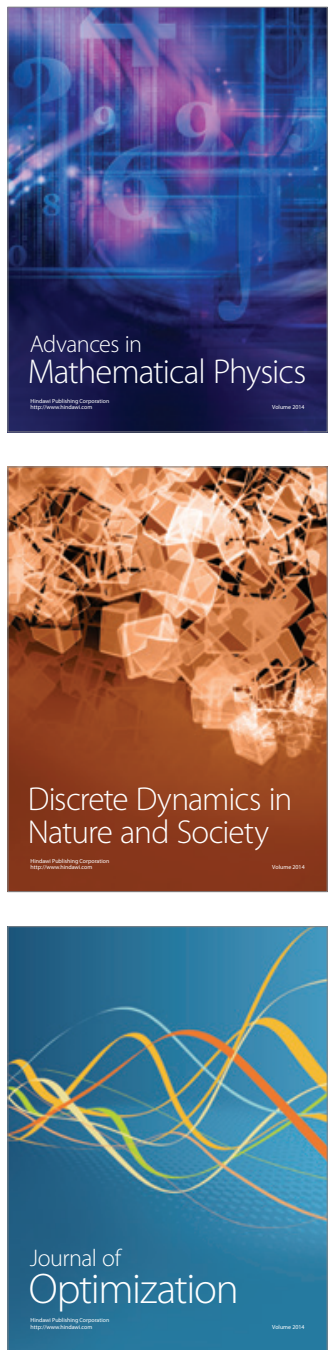